\title{
Der Bhopal-Case - Juristische Bewältigung des schlimmsten Industrieunfalls aller Zeiten
}

In der Nacht vom 2. auf den 3. Dezember 1984 ereignete sich in der indischen Stadt Bhopal ein folgenschwerer Industrieunfall. In einer Pestizidfabrik der Firma Union Carbide India Limited, einer Tochterfirma des amerikanischen multinationalen Konzerns Union Carbide Corporation, strömte durch ein Leck in einem Chemikalientank das Gas Methylisocyanat aus, das Bhopal in eine tödliche Wolke hüllte. Bis heute starben über 3500 Menschen, 25000 Bewohner von Bhopal erlitten zum Teil schwere Verletzungen an Augen, Atemwegen und Nervensystem. Viele leiden noch heute an den Folgen des Unfalls. Betroffen waren vor allem die Ärmsten der Armen, die sich, geduldet von der Regierung, in der Nähe der Fabrik in Slums angesiedelt hatten. ${ }^{\text {. }}$

Die juristische Auseinandersetzung um die Verantwortlichkeit von Union Carbide für das Unglück wurde vor amerikanischen und indischen Gerichten geführt. Nach mehr als vier Jahren wurde der Bhopal-Case durch einen Vergleich vor dem Supreme Court of India am I4. Februar 1989 abgeschlossen. ${ }^{2}$ Union Carbide verpflichtete sich, 470 Millionen US-Dollar an die Opfer zu zahlen. Dieser Vergleich hat in Indien zu heftigen, ablehnenden Reaktionen geführt. Er wurde vielfach als Kapitulation vor dem westlichen multinationalen Konzern verstanden.

In Europa hat die gerichtliche Auseinandersetzung um das Unglück von Bhopal zu Unrecht weit weniger Aufmerksamkeit erregt als der Unfall selbst, der 1984 vielfach als Beispiel für die Ausbeutung der Dritten Welt durch westliche Industrieunternehmen angesehen wurde. Erst jetzt läßt sich beurteilen, ob die Opfer Bhopals sich wenigstens vor den Gerichten gegenüber dem übermächtigen multinationalen Konzern durchsetzen konnten. Eine Betrachtung des Bhopal-Case ist außerdem deshalb von Interesse, weil dabei schwierige Rechtsfragen im Zusammenhang mit der Haftung von multinationalen Unternehmen bei Unfällen in der Dritten Welt auftauchten.

\section{I. "Ambulance Chasers" und Bhopal Gas Act $1985^{3}$}

Viele in Schadensersatzprozessen in den USA erfolgreiche Rechtsanwälte sahen in dem Unfall in Bhopal die große Chance, vor amerikanischen Gerichten enorme Schadensersatzforderungen gegen Union Carbide durchzusetzen. Die in den USA üblichen Erfolgshonorare für Rechtsanwälte ließen eine Vertretung der Opfer Bhopals als überaus lukrativ erscheinen. Deshalb trafen kurz nach dem Unfall amerikanische Rechtsanwälte in Bhopal ein, um einige der Opfer, häufig Analphabeten, mit dubiosen Methoden dazu zu bewegen, ihnen Vollmachten für Prozesse in den USA auszustellen.

I In der deutschen Presse wurden die Ereignisse in Bhopal am 2./3. 12. 1984 insbesondere im SPIEGEL $1984, \mathrm{Nr}$. $50 / 5 \mathrm{I}$ beschrieben.

2 Ich war zur Ableıstung meiner Referendar-Wahlstation von Februar bis Mai 1989 für die DeutschIndische Handelskammer in Neu Delhı tätı und konnte so die Diskussion um den Vergleich im BhopalCase verfolgen.

3 Quelle für die nachfolgend unter I-III geschilderte Vorgeschıchte des Vergleıchs war das Buch »BhopalThe lessons of a tragedy« von Sanjoy Hazarika, erschienen 1987. 
Um eine Übervorteilung der Opfer durch diese sogenannten »ambulance chasers« zu verhindern, erließ die indische Regierung im Februar 1985 den Bhopal Gas Act, der die indische Regierung zur alleinigen Repräsentantin der Opfer vor Gericht erklärte. Dieses Gesetz galt aber natürlich nur für Prozesse vor indischen Gerichten.

\section{Prozeß in Indien oder in den USA?}

Neben den amerikanischen Rechtsanwälten hatte aber auch die indische Regierung ein großes Interesse daran, daß der Prozeß gegen Union Carbide in den USA geführt wird.

Amerikanische Gerichte hatten in der Vergangenheit weit höhere Schadensersatzsummen zugesprochen als indische Gerichte in vergleichbaren Fällen. Die Rechtsprechung von US-Gerichten zum Nachweis des Verschuldens bei Industrieunfällen war für die Opfer vorteilhafter als die bisherige Praxis vor indischen Gerichten. Indische Gerichte hatten außerdem nur Zugriff auf das Vermögen der indischen Tochterfirma Union Carbide India Limited und nicht auf das Vermögen von Union Carbide. Die indische Regierung entschloß sich daher, Union Carbide in den USA $\mathrm{zu}$ verklagen. Ihre Klage wurde mit den in verschiedenen Städten der USA von privaten Rechtsanwälten erhobenen Klagen verbunden und am 6.2.1985 an das New Yorker Distriktgericht verwiesen. Zuständig wurde Richter Kennan. Union Carbide wollte aus den eben beschriebenen Gründen unter allen Umständen verhindern, daß der Prozeß in den USA stattfindet, so daß der Kernpunkt der Auseinandersetzung vor Richter Kennan die Frage des Gerichtsstandes war.

Der Richter war bei dieser Frage, wie in weiten Bereichen des anglo-amerikanischen Rechts, nicht an gesetzliche Vorgaben gebunden, sondern konnte den Fall, orientiert an eventuellen Präzedenzfällen, frei entscheiden.

Union Carbide begründete ihre Auffassung von der Unzuständigkeit des New Yorker Gerichts damit, daß der Unfall in Indien passiert sei und sämtliche Beweismittel sich in Indien befänden, so daß der Prozeß dort stattfinden müsse.

Die indische Regierung betonte demgegenüber, daß die Beklagte eine amerikanische Firma sei, und führte außerdem aus, daß die indische Justiz nicht in der Lage sei, den Fall befriedigend zu lösen. Außerdem sei es zweifelhaft, ob Union Carbide dem Urteil eines indischen Gerichts ohne weitere rechtliche Schritte in den USA Folge leisten würde. Union Carbide verteidigte daraufhin das indische Rechtssystem gegen die indische Regierung und legte Aussagen von namhaften indischen Juristen vor, welche die Effizienz indischer Gerichte beweisen sollten.

Richter Kennan versuchte erfolglos, einen Vergleich zu erreichen. Die indische Regierung war nicht bereit, das Angebot von Union Carbide, zuletzt angeblich 300 Millionen US-Dollar, zu akzeptieren.

In seinem Urteil vom I2. 5. 1986 erklärte sich Richter Kennan für unzuständig. Er schloß sich der Auffassung von Union Carbide an, daß der Prozeß in Indien stattfinden müsse, da sich sämtliche Beweismittel dort befänden. Die von der indischen Regierung vorgetragene mangelnde Effizienz des indischen Rechtssystems ließ Richter Kennan nicht als Argument für einen Prozeß in den USA gelten. Zum einen war er entgegen der indischen Regierung der Auffassung, daß die indischen Gerichte einen solchen Fall befriedigend lösen könnten. Zum anderen führte Richter Kennan aus, daß eine mit der Unfähigkeit des indischen Rechtssystems begründete Bejahung der amerikanischen Gerichtsbarkeit nichts anderes als Imperialismus wäre. 
Die Berufung der indischen Regierung gegen dieses Urteil wurde in den USA verworfen.

III. Der Bhopal-Case vor dem Bhopal Distruct Court und dem Madya Pradesh High Court

Am 5.9. 1986 erhob die indische Regierung, die nunmehr aufgrund des bereits erwähnten Bhopal Gas Act die alleinige Repräsentantin der Opfer war, Klage gegen Union Carbide vor dem Bhopal District Court. Es wurde ein Schadensersatzanspruch in Höhe von 3, I Milliarden US-Dollar geltend gemacht.

Nachdem die Vorfrage des Gerichtsstandes bisher im Mittelpunkt der Auseinandersetzung gestanden hatte, ging es nun um die eigentlichen Rechts- und Tatsachenfragen.

Entscheidende, über den konkreten Fall hinausgehende Rechtsfrage wurde, inwieweit ein multinationales Unternehmen für Schäden, die durch eine Tochterfirma in der Dritten Welt verursacht wurden, haftet. Eine solche Haftung lehnte Union Carbide ab. Union Carbide verstand ihre Tochterfirma Union Carbide India Limited, die die Fabrik in Bhopal betrieb, als unabhängige Gesellschaft, für deren Tun und Unterlassen die Muttergesellschaft nicht einzustehen habe. Die indische Regierung hielt dem entgegen, daß ein multinationales Unternehmen durch die straffe Führung der Tochtergesellschaften durch die Muttergesellschaft gekennzeichnet sei. Sämtliche wichtigen Entscheidungen für die Fabrik in Bhopal seien von der Konzernspitze in den USA abgesegnet worden. Technologie und Sicherheitseinrichtungen seien von der Muttergesellschaft geliefert und ständig überprüft worden. Die Struktur von multinationalen Unternehmen, ein Netzwerk von Muttergesellschaft und verschiedenen Tochtergesellschaften, mache es dem einzelnen von einem Unfall betroffenen Bürger unmöglich, festzustellen, welcher Teil des multinationalen Konzerns für den Unfall verantwortlich sei. Deshalb müsse die Muttergesellschaft für die Folgen von Unfällen in Fabriken ihrer Tochtergesellschaften haften.

Auch die Ursache des Unfalls blieb umstritten. Während nach Auffassung der indischen Regierung verschiedene Versäumnisse der Firmenleitung bei der Wartung des Chemikalientanks und bei den Sicherheitseinrichtungen den Unfall herbeigeführt hatten, behauptete Union Carbide, das Leck in dem Chemikalientank sei durch Sabotage entstanden, ohne jedoch die Umstände der Tat und die Identität des Täters offenzulegen.

Ein weiterer Schwerpunkt in der Prozeßstrategie von Union Carbide war die Behauptung eines Mitverschuldens der indischen Bundesregierung und der Regierung des indischen Bundesstaates Madya Pradesh, in dem Bhopal liegt. Der Sicherheitsstandard der Fabrik sei von Regierungsbeamten mehrmals ohne jegliche Beanstandung überprüft worden. Die Regierung habe außerdem illegale Siedlungen in der Nähe der Fabrik geduldet und somit zum Ausmaß der Katastrophe beigetragen.

Wegen der komplizierten Sach- und Rechtslage rechnete man mit einem jahrelangen Rechtsstreit. Vergleichsverhandlungen scheiterten wiederum an dem Widerstand der indischen Regierung. Da die Opfer auf eine schnelle Hilfe angewiesen waren, erließ Richter Deo vom Bhopal District Court eine einstweilige Verfügung, in der Union Carbide zur Zahlung von 350 Millionen Rupien (ca. 44 Millionen DM) verurteilt wurde. Auf die Berufung von Union Carbide gegen diese einstweilige Verfügung wurde dieser Betrag vom Madya Pradesh High Court auf 250 Millionen Rupien (ca. 3 I Millionen DM) herabgesetzt. 
Union Carbide und die indische Regierung legten gegen diese Entscheidung Rechtsmittel zum Supreme Court of India ein.

\section{Vergleich vor dem Supreme Court of India}

Obwohl es in dem Verfahren vor dem Supreme Court nur um die Berechtigung der einstweiligen Verfügung ging, kam es am I 4. 2. I989 nach vier Monaten umfassender Erörterung der Probleme des Falles zur Überraschung der Öffentlichkeit zu einem auf die endgültige Beilegung des Rechtsstreites gerichteten Vergleichsvorschlag des Gerichtes, der sofort von beiden Seiten akzeptiert wurde.

Union Carbide verpflichtete sich zur Zahlung von 470 Millionen US-Dollar als Schadensersatz für alle aus dem Unfall entstandenen Schäden. Zusätzlich wurden in dem Vergleich sämtliche strafrechtlichen Verfahren gegen Angestellte von Union Carbide für beendet erklärt. ${ }^{4}$

Dieser Vergleich, dem offensichtlich außergerichtliche Verhandlungen vorausgegangen waren, löste in der indischen Öffentlichkeit Empörung aus.' Die Presse, die Oppositionsparteien und die Organisationen der Opfer hielten den vereinbarten Betrag von 470 Millionen US-Dollar für absolut unzureichend. Mit Bitterkeit wurde darauf hingewiesen, daß ein amerikanisches Gericht eine weit höhere Schadensersatzsumme festgesetzt hätte. Dem Supreme Court wurde deshalb der Vorwurf gemacht, das Leben der Opfer von Bhopal für weniger wert zu halten als das Leben von Amerikanern.

Scharf kritisiert wurde, daß die Opfer von Bhopal bzw. deren Organisationen vor dem Abschluß des Vergleichs in keiner Weise konsultiert worden waren.

Juristen bezweifeln, daß der Supreme Court, der ja nur mit der einstweiligen Verfügung befaßt war, überhaupt berechtigt war, einen auf die endgültige Beendigung der Hauptsache gerichteten Vergleichsvorschlag zu machen. Auch die Niederschlagung aller strafrechtlichen Verfahren in einem zivilrechtlichen Vergleich wurde von namhaften Juristen für unzulässig gehalten.

Die indische Regierung verteidigte den Vergleich mit der Notwendigkeit einer schnellen Entschädigung der Opfer. Die endgültige Entscheidung des Rechtsstreits hätte wegen der verschiedenen Rechtsmittelmöglichkeiten in Indien und den USA hier mußte ein letztinstanzliches Urteil gegen Union Carbide vollstreckt werden bis zu 20 Jahren dauern können. Die Hilfe würde dann zu spät kommen. Weiterhin wurde zur Verteidigung des Vergleichs angeführt, daß wegen der entscheidenden Rechtsfrage, ob multinationale Konzerne für Versäumnisse ihrer Tochtergesellschaften haften, ein erhebliches Prozeßrisiko bestanden hätte.

\section{Fazit}

Union Carbide hat sich mit seinen beiden Hauptzielen, den Prozeß vor einem indischen und nicht einem amerikanischen Gericht durchzuführen und den Fall vergleichsweise, d. h. ohne richterliche Feststellung einer Verantwortlichkeit für das Unglück, zu beenden, durchgesetzt.

Die Schadensersatzsumme von 470 Millionen US-Dollar ist erheblich geringer als der Betrag, den ein amerikanisches Gericht als Schadensersatz zugebilligt hätte, und

4 Der Vergleıch ist in der März-Ausgabe der indischen Zeitschrift »The Law Magazıne « 1989, Seiten 36/37 abgedruckt.

5 Die $1 \mathrm{~m}$ Folgenden dargestellte Kritik des Vergleıchs habe ıch den indischen Zeitungen "Times of India «, "Hindustan Times", "Indian-Express" vom I 5.-25. I. 1989 entnommen. Kritik von Juristen fand sich außerdem in der März-Ausgabe von "The Law Magazıne«. 
liegt erheblich unter dem Betrag, den die indische Regierung zunächst gefordert hatte. Die Opfer konnten die Vergleichssumme deshalb nur als unzureichend empfinden.

Sicher ist es zutreffend, daß das Durchfechten des Prozesses in der Hauptsache mit den verschiedenen Rechtsmittelmöglichkeiten in Indien und den USA eine jahrelange Prozeßdauer mit ungewissem Ausgang zur Folge gehabt hätte. Durch die Aufrechterhaltung der einstweiligen Verfügung und weitere Zahlungen der Regierung hätte den Opfern aber eine vorläufige Hilfe geboten und der Prozeß in der Hauptsache fortgeführt werden können. Das Argument der Regierung, nur der Vergleich habe eine rechtzeitige Entschädigung der Opfer ermöglicht, verliert auch deshalb an Überzeugungskraft, weil die indische Regierung vor dem New Yorker Distriktgericht und dem Bhopal District Court einen Vergleich strikt abgelehnt hatte. Wenn man ein langwieriges Gerichtsverfahren hätte vermeiden wollen, so hätte der Vergleich schon vor Richter Kennan oder spätestens vor dem Bhopal District Court, nicht aber mehr als 4 Jahre nach dem Unglück abgeschlossen werden müssen.

Die indische Regierung, die sich durch den Bhopal Gas Act zur alleinigen Repräsentantin der Opfer gemacht hatte, ist ihrer damit verbundenen Verantwortung durch diese mangelhafte Prozeßführung nicht gerecht geworden.

Die Kombination von verantwortungslosem Unternehmertum und staatlicher Inkompetenz hat nicht nur den Unfall verursacht, sondern auch eine gerechte Entschädigung der Opfer von Bhopal verhindert.

Aus der Sicht des Juristen ist zu bedauern, daß wichtige Rechtsfragen im Zusammenhang mit der Tätigkeit von multinationalen Unternehmen in der Dritten Welt nicht befriedigend geklärt wurden. Wegen des Vergleichs wurde die wichtige Rechtsfrage, ob multinationale Unternehmen für Versäumnisse in Fabriken ihrer Tochterfirmen haften, nicht entschieden. Es wurde versäumt, einen Präzedenzfall zu setzen, der multinationale Konzerne ermahnt hätte, in der Dritten Welt den gleichen Sicherheitsstandard zu erfüllen wie in den westlichen Industrienationen.

Auch die Entscheidung von Richter Kennan zum Gerichtsstand bei Prozessen gegen multinationale Konzerne bei Unfällen in der Dritten Welt ist unbefriedigend. Die Verneinung einer Klagemöglichkeit im Heimatland des multinationalen Unternehmens führt dazu, daß sich die Haftung des multinationalen Unternehmens nach den meist weniger strengen Maßstäben der Gerichte in der Dritten Welt richtet. Der geringe Lebensstandard in den Ländern der Dritten Welt hat zur Folge, daß die Schadensersatzsumme bei Bejahung einer Haftung des multinationalen Konzerns erheblich geringer sein wird als bei einem Prozeß in Europa oder den USA.

Nur bei Einräumung einer Klagemöglichkeit in den Heimatländern der multinationalen Unternehmen werden diese bereit sein, in ihren Fabriken der Dritten Welt für den gleichen Sicherheitsstandard zu sorgen wie in ihren Fabriken in Europa oder den USA.

Insgesamt bleibt festzuhalten, daß der Bhopal Case mit einem für die Opfer unbefriedigenden Ergebnis endete und die Möglichkeit, für die Zukunft Maßstäbe für die Aktivitäten multinationaler Konzerne in der Dritten Welt zu setzen, nicht wahrgenommen wurde. 